\title{
Requisitos básicos para los sistemas de registro y gestión de dosis en pacientes sometidos a exploraciones de diagnóstico por imagen
}

\section{Basic requirements for systems of dose management and recording in imaging diagnostic examinations}

\author{
Santiago Miquelez Alonso ${ }^{1}$, Miguel Ángel Peinado Montes ${ }^{2}$, José Miguel Fernandez Soto ${ }^{3}$, \\ José Manuel Ordiales Solís ${ }^{4}$, Luis Alejo Luque ${ }^{5}$, Julio Fco. Almansa López ${ }^{6}$, Joan Font Gelabert ${ }^{7}$ \\ 1 Jefe de Servicio de Radiofísica y P.R. (en funciones). Complejo Hospitalario deNavarra. \\ 2 Jefe de Servicio del Hospital Universitario Central de Asturias. \\ 3 Jefe del Servicio de Física Médica (en funciones). Hospital Clínico San Carlos. \\ 4 Jefe del Servicio de Radiofísica, Hospital de Mérida. \\ 5 F.E.A Servicio de Radiofísica y Radioprotección Hospital Universitario de la Paz \\ 6 FEA Radiofisica Hospitalaria. Hosp. Universitario Virgen de las Nieves. \\ 7 Jefe del Servicio de Radifísica Hospitalaria. Hospital Universitari de Son Espasses.
}

Fecha de Recepción: 11/12/2019 - Fecha de Aceptación: 10/03/2020

La Directiva 2013/59/Euratom del Consejo de 5 de diciembre de 2013 por la que se establecen normas de seguridad básicas para la protección contra los peligros derivados de la exposición a radiaciones ionizantes, establece en el apartado 28 de la introducción que se "debe proponer requisitos más estrictos en cuanto a la información que debe proporcionarse a los pacientes, el registro y la notificación de las dosis de los procedimientos médicos, el uso de niveles de referencia para diagnóstico y la disponibilidad de dispositivos indicadores de dosis". Ante el desarrollo de estos conceptos en el citado documento y la posterior trasposición a la ley española mediante el Real Decreto 601/2019, de 18 de octubre, sobre justificación y optimización del uso de las radiaciones ionizantes para la protección radiológica de las personas con ocasión de exposiciones médicas, la Sociedad Española de Protección Radiológica (SEPR) y la Sociedad Española de Física Médica (SEFM) han creado un grupo de trabajo para describir, mediante este informe, los requisitos que debe cumplir un sistema de registro y gestión de dosis.

Palabras clave: dosis al paciente, registro de dosis, optimización, niveles de referencia para diagnóstico, dispositivos indicadores de dosis.

Directive 2013/59/Euratom of 5 December 2013 laying down basic safety standards for protection against the dangers arising from exposure to ionising radiation, establishes in section 28 of its introduction that more strict requirements should be proposed in what information to patients, recording and notification of doses in medical procedures, use of reference levels for diagnosis (RLD) and availability of dose indicator devices concerns. Due to the development of said concepts in both the European and Spanish regulation (Real Decreto 601/2019 of 18 October 2019 about justification and optimization of the use of ionising radiation for radiological protection in medical exposures) the Spanish Societies of Radiological Protection (SEPR) and Medical Physics (SEFM) have created a work group whose task is to make a report describing the requirements that a system for the recording and management of doses should fulfil.

Key words: dose recording, optimization, reference levels for the diagnosis, dose indicator devices.

\footnotetext{
*Correspondencia: santiago.miquelez.alonso@navarra.es https://doi.org/10.37004/sefm/2020.21.1.004
} 


\section{Tabla de contenido}

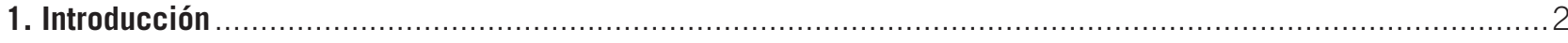

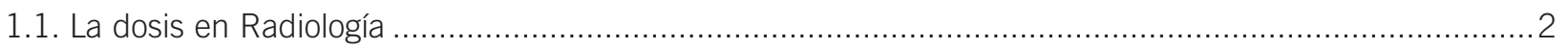

1.2. "Si no puede medirse, no puede mejorarse" ............................................................................ 3

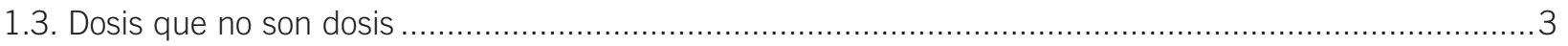

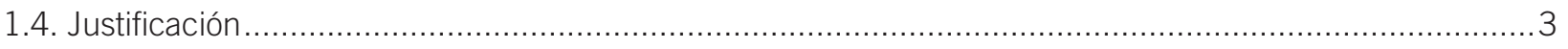

2. Optimización y uso de los niveles de referencia para diagnóstico ........................................................ 4

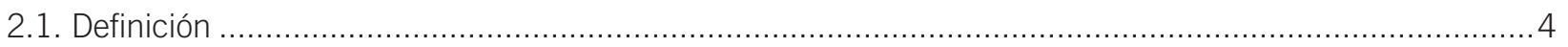

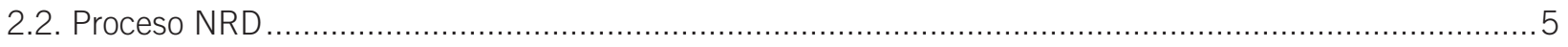

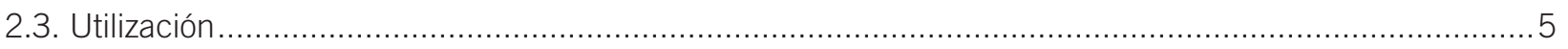

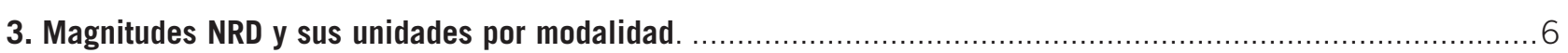

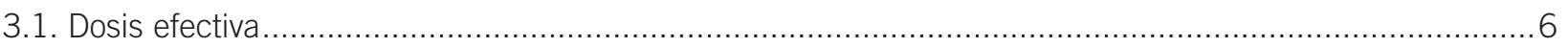

4. Requisitos de los sistemas de registro de indicadores dosimétricos ................................................ 7

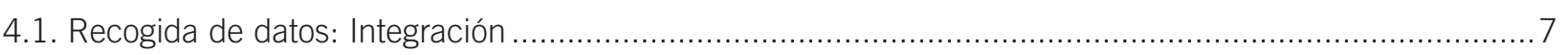

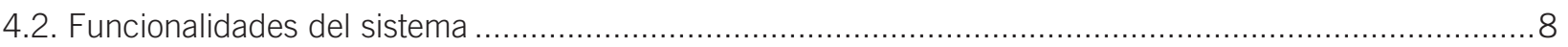

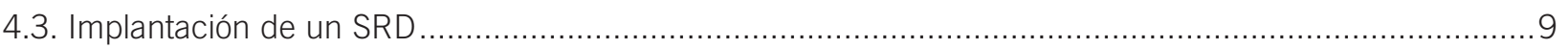

5. Verificación (control de calidad) de las prestaciones del sistema de registro de dosis................................ 10

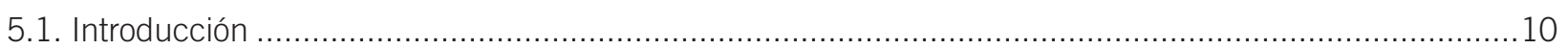

5.2. Procesos que intervienen en la gestión de la dosis ............................................................... 10

5.3. El Protocolo Español de Control de Calidad en Radiodiagnóstico Revisión 2011 (PECCRX)...................10

6. Responsabilidades del radiofísico hospitalario en los sistemas de gestión de dosis ...................................11

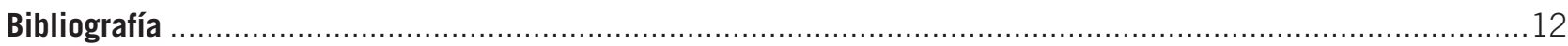

\section{Introducción}

\subsection{La dosis* en Radiología}

La dosis impartida a los pacientes, debido a las radiaciones ionizantes, ha sido una de las principales preocupaciones de los profesionales de la Radiología. ${ }^{\dagger}$

Sin embargo, esta "mentalidad de la dosis" ha ido tomando una mayor relevancia en estos últimos años en los que estudios retrospectivos ${ }^{1,2,3}$ anunciaban resultados alarmantes y las previsiones predecían un panorama aún peor. ${ }^{4,5}$

Esta concienciación se ha concretado en algunas iniciativas conjuntas como Image Gently, ${ }^{6}$ en pacien-

\footnotetext{
*A lo largo de este informe, se utiliza de modo genérico el término simplificado de "dosis" para designar algunas magnitudes y usar determinadas expresiones, habituales en las áreas del radiodiagnóstico y radioprotección. Entre las magnitudes, las ligadas a la dosis absorbida y las dosimétricas específicas del radiodiagnóstico y las inherentes a la radioprotección. Entre las expresiones, se pueden citar: dosis de radiación, dosis en el paciente, dosis impartida al paciente, etc.

${ }^{\dagger}$ En sentido amplio, incluyendo todas las especialidades que realizan procedimientos diagnósticos o intervencionistas.
}

tes pediátricos, o Image Wisely en adultos. Dichas iniciativas inciden particularmente en la necesidad de optimizar el acto radiológico para asegurar que se está utilizando solo la cantidad necesaria de radiación para producir imágenes adecuadas al objetivo diagnóstico.

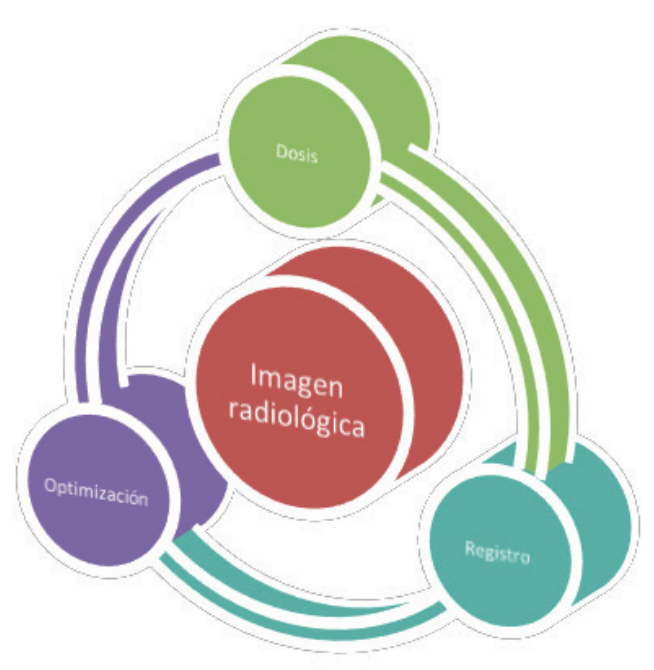




\section{2. "Si no puede medirse, no puede mejorarse"}

En el ámbito de la Radiofísica, las dosis recibidas por los pacientes se vienen midiendo desde hace más de 60 años. Aunque dichas medidas se llevan a cabo individualmente, la dificultad de la puesta en marcha del dispositivo experimental hace inviable una determinación individual de la dosis absorbida de forma sistemática.

Por el contrario, su objetivo siempre ha sido la determinación de promedios estadísticos en distintos ámbitos (local, nacional o regional) y en última instancia el establecimiento de los niveles de referencia diagnósti$\cos \left(N_{R D} 8,9,10,11,12\right)$ que se emplean como un indicador de calidad para la optimización de los procedimientos radiológicos.

Cabe destacar el progreso que ha supuesto en este ámbito la digitalización de la imagen, por cuanto ha permitido recoger, cada vez de forma más exhaustiva, toda la información que es relevante a la hora de estimar las dosis de radiación. Se dispone entonces de un volumen de información que permitirá extraer resultados de indicadores de dosis, tanto poblacionales como individuales.

\subsection{Dosis que no son dosis}

Se plantea entonces una serie de problemas adicionales que se han ido resolviendo con mayor o menor fortuna:

- Qué información recoger: Si bien se sabe qué parámetros influyen en la dosis recibida por el paciente y cómo calcular dicha dosis, ha de tenerse en cuenta que no se están midiendo dosis absorbidas directamente.

- Cómo codificarla: Tanto NEMA dentro de su norma DICOM, ${ }^{13}$ como IHE con sus perfiles REM y REM-M, han definido la estructura de dicha información.

- Cómo convertir esa información en dosis: El paso de los parámetros almacenados por las modalidades a la dosis absorbida por el paciente, que es la magnitud física principal, no es simple ni desde luego exacto a nivel individual. Los métodos actuales se basan por lo general en simulaciones de Montecarlo que contemplan pacientes estándar en lo referente a tamaño y distribución de órganos.

En lugar de la dosis, generalmente se emplean otras magnitudes que están relacionadas con ésta (como el

${ }_{\mp \text { Sir William Rutherford. }}$
CTDI para la tomografía computarizada o la dosis de entrada en piel en fluoroscopia) o se calcula en unas condiciones más o menos normalizadas (como la dosis glandular promedio en el caso de la mamografía).

En cualquier caso, haciendo de la necesidad virtud, puesto que no necesariamente se necesita conocer dicha dosis absorbida, tanto si se va a emplear dicha información para establecer NRD, para monitorizar la cantidad de dosis de radiación impartida o para definir cualquier tipo de registro individual, se puede definir prácticamente todo el análisis a partir de esos "sustitutos" conocidos como indicadores de dosis.

\subsection{Justificación}

Aún siendo el otro pilar sobre el que se sustenta la protección radiológica de los pacientes en el ámbito de las exposiciones médicas, el esfuerzo realizado en la optimización no se ha visto, sin embargo, acompañado por uno similar en el ámbito de la justificación.

Las razones de esta deficiencia quizás tienen que ver con motivos históricos, puesto que el impacto (beneficio) de la imagen en el curso del tratamiento de los pacientes era más que evidente y no ha sido hasta la "explosión" de la utilización de la radiología en los últimos 30 años que ésta se ha convertido en un tema de preocupación.

Es en este momento cuando algunos autores han puesto de manifiesto que, según las exploraciones, puede haber entre un 20\% y un $50 \%$ de pruebas cuya indicación puede ser inadecuada. ${ }^{14}$

Las acciones encaminadas a corregir este déficit han consistido en la edición de guías de indicación y recomendaciones de "no hacer",15,16 aunque nuestra experiencia apunta a que su impacto en la práctica clínica ha sido muy pequeño, por no decir irrelevante.

La conclusión principal es la necesidad de insistir en:

1. La concienciación (y el conocimiento) de profesionales y pacientes sobre las dosis de radiación y sus riesgos.

2. La adecuación de las pruebas diagnósticas solicitadas a la patología.

3. La auditoría periódica del proceso radiológico para encontrar oportunidades de mejora en esos aspectos. ${ }^{15}$

La aparición de los software de gestión de dosis, junto con los necesarios procesos de integración con el PACS y el RIS, permite avanzar en los tres aspectos citados, ${ }^{17}$ siendo el momento adecuado de introducir esta etapa de justificación en el flujo de peticiones de 
exploraciones radiológicas. Esto redundará no sólo en la reducción de la dosis colectiva y en la seguridad del paciente, sino también en un uso más eficiente de los recursos de los Servicios de Radiología. ${ }^{18}$

\section{Optimización y uso de los niveles de referencia para diagnóstico}

Desde su aparición en $1991^{19}$ hasta la publicación del último informe específico ${ }^{20}$ la ICRP (Comisión Internacional de Protección Radiológica, de sus siglas en inglés) considera los niveles de referencia para diagnóstico (NRD) una herramienta clave para la optimización.

Abundando en el papel central que juegan estos NRD, la Directiva Europea 2013/59/EURATOM ${ }^{21}$ incide en la obligación de adoptar sin dilación las medidas correctoras adecuadas, siempre que dichos niveles se superen de manera sistemática.

Con objeto de responder adecuadamente al requerimiento legal, las sociedades científicas implicadas (SEFM, SEPR y SERAM) han acordado la elaboración de una "Guía para la Optimización y uso de los Niveles de Referencia para Diagnóstico" que facilite a los profesionales implicados la utilización correcta de la tecnología que permita llevar a cabo las acciones necesarias en aquellos procedimientos diagnósticos o intervencionistas que no estén optimizados.

A continuación se definen los NRD, su alcance, los parámetros estadísticos que mejor describen a las poblaciones y el proceso para su determinación y uso en la optimización.

Las definiciones empleadas a continuación son las que aparecen en el documento "Diagnostic reference levels in medical imaging. ICRP Publication 135". ${ }^{12}$ Así mismo, este documento sirve de guión para el desarrollo del capítulo.

\subsection{Definición}

El NRD se define como un nivel de investigación establecido en términos de un valor de un indicador de dosis que es utilizado como herramienta de optimización de las exposiciones médicas en procedimientos radiológicos e intervencionistas.

\subsubsection{Indicador de dosis}

Es la magnitud empleada en los NRD. Se utiliza un parámetro de uso común fácil de medir o calcular para la modalidad de que se trate y que evalúa la cantidad de radiación ionizante utilizada para obtener las imágenes médicas.
Con excepción de la dosis glandular media utilizada en mamografía, los indicadores de dosis no son dosis absorbidas en órganos o tejidos del paciente ni cantidades derivadas, ya que estas "dosis" no pueden ser medidas o calculadas fácilmente. Por razones similares, tampoco se aconseja el uso de la magnitud dosis efectiva.

En caso de que se utilicen dos modalidades de imagen para el mismo procedimiento (por ejemplo, PET-CT, SPECT-CT), se deben presentar los indicadores de ambas modalidades de manera independiente.

\subsubsection{Valor del NRD}

El valor de un NRD se establece como el tercer cuartil de una distribución de medianas que han sido obtenidas de las distribuciones de los indicadores de dosis observadas en distintos centros.

Los valores de los NRD deben obtenerse de distribuciones de indicadores de dosis por procedimiento en una muestra apropiada de pacientes. El uso de maniquís no es suficiente, ya que en ese caso no se tiene en cuenta el posible efecto producido por la manipulación del equipo por parte del operador.

\subsection{3. Ámbito}

En función del número de centros involucrados en la determinación del valor del NRD éste puede clasificarse en tres niveles sucesivos, a saber:

a. Locales: Son NRD establecidos para cada procedimiento clínico en un grupo de salas o instalaciones dentro de un país. Estarán calculados a partir del tercer cuartil de la distribución de las medianas de los indicadores de dosis correspondientes.

Pueden obtenerse en procedimientos para los que no existen NRD nacionales o regionales, o también para los casos en los que aun existiendo dichos NRD, el equipamiento local o las técnicas aplicadas permiten conseguir un mayor grado de optimización, de manera que pueda implementarse un valor del NRD menor que el correspondiente al NRD nacional o regional.

b. Nacionales: Valores de NRD establecidos por país y procedimiento. Están calculados como el valor del tercer cuartil de la distribución de las medianas de los indicadores de dosis obtenidos en cada centro médico de una muestra representativa de instalaciones. 
c. Regionales : Establecidos por procedimiento clínico en una única región, basados en la mediana de una muestra representativa de valores de NRD nacionales.

Se definen además, los valores típicos como los correspondientes a la mediana de la distribución de los indicadores de dosis en un centro o pequeño grupo de centros. Son útiles para fomentar procesos de optimización.

Se requiere cierta flexibilidad en los requisitos estadísticos para el establecimiento de los NRD en procedimientos en los que se dispone de pocos datos, como por ejemplo en radiología intervencionista pediátrica, o cuando los datos que se disponen provienen de pocos centros.

\subsection{Proceso NRD}

Se denomina así al proceso cíclico de determinación de valores de NRD actualizados para que puedan ser utilizados como herramientas para la optimización.

Es de resaltar que la aplicación de este proceso no es suficiente por sí mismo para la completa realización de un procedimiento de optimización dosimétrica.

Ya que tanto la tecnología implicada como los procedimientos radiológicos están en continuo proceso de mejora y adaptación, los valores de los NRD deben actualizarse regularmente.

Los NRD nacionales o regionales deben ser revisados con una periodicidad no superior a los 5 años, o incluso más frecuentemente en caso de que se produzca una mejora sustancial en la tecnología o en el posprocesado de las imágenes, o bien se establezcan nuevos protocolos de imagen.

Además, en caso de que se introduzcan nuevas técnicas de imagen, deben obtenerse distribuciones suficientemente significativas de los indicadores de dosis y establecer los valores de los NRD tan pronto como sea posible.

Finalmente, la mejor manera de evaluar la efectividad de los NRD es estudiando su evolución en el tiempo, y resulta por tanto necesario realizar análisis de tendencias.

\subsection{Utilización}

Los NRD se utilizan en imagen médica realizada con radiación ionizante para indicar si en la práctica habitual de un determinado procedimiento radiológico o intervencionista la cantidad de radiación aplicada es

\footnotetext{
§Nótese que el término regional difiere del comúnmente aceptado en español, correspondiendo aquí a un ámbito supranacional. En nuestro caso concreto se refiere a Europa.
}

inusualmente alta o baja para ese tipo de procedimiento (Optimización).

Una vez determinada la mediana de la distribución de los indicadores de dosis correspondientes a la modalidad, por procedimiento y sala radiológica, deben ser comparadas con los valores de los NRD locales, nacionales o regionales. Dicha distribución debe obtenerse para una muestra representativa de pacientes de un rango apropiado de edad o peso.

Si dicho valor excede al NRD correspondiente o es muy inferior a éste, se debe realizar lo antes posible una investigación para determinar las causas. En esta investigación debe evaluarse también la calidad de las imágenes obtenidas, o de manera más general, la información diagnóstica proporcionada por el examen (incluyendo los efectos del posprocesado). Se recomienda establecer unos criterios básicos de calidad de imagen clínica, basados en la puntuación de indicadores de calidad adecuados para los procedimientos principales. Esto posibilitará la puntuación objetiva de la calidad de la imagen clínica. Los test realizados con maniquíes pueden servir como referencia a la hora de detectar cambios en el equipamiento empleado.

En el primer caso, la optimización trata de mantener la calidad de la información diagnóstica proporcionada por el examen, de acuerdo con el propósito médico, mientras que al mismo tiempo busca reducir la exposición del paciente a la radiación a un nivel tan bajo como sea razonable conseguir. En el segundo caso, el procedimiento de optimización puede requerir un aumento de la dosis de radiación si se detecta que la calidad de la información diagnóstica obtenida es insuficiente.

Si se requieren acciones correctivas, se debe documentar e implementar sin dilación un plan de acción en la sala que incluya tanto un ajuste de los parámetros de la exposición como un análisis de la calidad de la imagen.

En el proceso de optimización, coordinado por el especialista en Radiofísica Hospitalaria, deberán estar implicados radiólogos y médicos nucleares, así como otros especialistas médicos que emplean procedimientos diagnósticos o intervencionistas, los técnicos superiores en diagnóstico por imagen y los técnicos de las empresas responsables del mantenimiento y de las reparaciones de los equipos.

\subsubsection{Cómo no se deben emplear los NRD}

Los límites de dosis no se aplican a pacientes sometidos a exposiciones médicas. Los NRD son una herramienta esencial en los procesos de optimización, precisamente debido a este motivo. Es pues importante resaltar que los NRD no constituyen un límite de dosis para los pacientes. 
También hay que tener siempre en cuenta que los NRD no se deben aplicar a pacientes individuales y que hay multitud de causas que justifican que individualmente puedan superarse éstos sin que eso signifique una técnica inadecuada.

\subsubsection{Consideraciones para pacientes pediátricos}

El establecimiento de valores de NRD en pacientes pediátricos es más difícil que para los adultos, debido a que los niños presentan unos tamaños mucho más variables. El peso de los niños, por ejemplo, puede variar en un factor de más de 100 de un prematuro a un adolescente obeso.

Esta misma variabilidad en peso y tamaño hace que la cantidad de radiación utilizada en los exámenes de niños pueda ser muy diferente de unos pacientes a otros. Sin embargo, esto no excluye la posibilidad de una selección incorrecta de las técnicas radiológicas, o a un fallo en la adaptación del protocolo de imagen al tamaño del paciente o al propósito clínico.

En conclusión, no debería utilizarse un único paciente estándar para definir valores de NRD en imagen pediátrica. Al contrario, se recomienda establecer rangos de peso para el establecimiento de los valores de los NRD: $<5 \mathrm{~kg}, 5-<15 \mathrm{~kg}, 15-<30 \mathrm{~kg}, 30-<50 \mathrm{~kg}$, y $50-<80 \mathrm{~kg}$. En caso de que sólo se disponga de datos de edad del paciente, se recomienda establecer rangos en forma de bandas agrupadas en torno de $<1$ mes, 1 mes- $<4$ años, $4-<10$ años, 10-<14 años y $14-<18$ años.

En caso de exámenes de cabeza, se recomienda utilizar rangos de edad en lugar de rangos de peso para establecer los valores de los NRD: $<3$ meses, 3 meses$<1$ año, $1-<6$ años $y \geq 6$ años. ${ }^{22}$

\section{Magnitudes NRD y sus unidades por modalidad}

Durante el capítulo anterior se ha incidido en la necesidad de establecer NRD y en la conveniencia de determinar sus valores no en función de la dosis absorbida por el paciente, sino de una magnitud relacionada con aquella pero cuya determinación sea fácil, bien mediante la captura de los factores de técnica de la exploración o bien mediante un cálculo sencillo a partir de aquellos.

A continuación se muestran los indicadores de dosis por modalidad recomendados, así como sus correspondientes unidades.

- Intervencionismo y fluoroscopia: Producto kermaárea $\left(P_{K A}\right),\left[m G y \cdot \mathrm{cm}^{2}\right]$; kerma en el punto de referencia a la entrada del paciente $\left(K_{a, r}\right)$, [mGy]; tiempo de fluoroscopia [s] y número de adquisiciones de cine.

- Tomografía computarizada: producto dosis-longitud (DLP) para el estudio completo, [mGy $\cdot \mathrm{cm}$ ]; índice de dosis volumétrico en TC (CTDIvol) para cada secuencia, [mGy]. El uso de la magnitud SSDE [mGy] proporciona estimaciones más precisas de la dosis en pacientes pediátricos. ${ }^{23}$

- Mamografía: dosis glandular media $\left(D_{G}\right)$, [mGy].

- Radiología convencional: producto kerma-área $\left(P_{K A}\right),\left[m G y \cdot \mathrm{cm}^{2}\right]$; kerma en aire en la superficie de entrada $\left(K_{a, e}\right)$, [mGy].

- Medicina nuclear: actividad administrada [MBq]; actividad administrada por peso del paciente $\left[\mathrm{MBq} \cdot \mathrm{kg}^{-1}\right]$ de un radiofármaco específico para un procedimiento clínico concreto.

En procedimientos multimodalidad (SPECT-CT, PET-CT), los valores de los NRD deben establecerse para cada modalidad independientemente. Además, en procedimientos digitales avanzados que realicen adquisiciones multimagen (como por ejemplo la Tomosíntesis, los TC de energía dual o los CBCT), deberían distinguirse estos procedimientos de los convencionales y establecerse NRD propios.

\subsection{Dosis efectiva}

Mención especial merece la obtención de la dosis efectiva. Dicha magnitud permite la "unificación" de todas las dosis obtenidas en las diferentes exploraciones (diferentes regiones anatómicas y en diferentes momentos) y permitir obtener unos valores sumables entre ellos. ${ }^{18}$

Sin embargo es necesario remarcar que, estrictamente hablando, la dosis efectiva es una magnitud sólo válida si se utiliza en la estimación de riesgos a la población en su conjunto ${ }^{20}$ y no debe emplearse para cuantificar el riesgo individual a partir del valor calculado para un paciente, ni debe utilizarse o incluirse en informes generados por el sistema de registro de dosis (SRD) sin la supervisión previa por parte del especialista en Radiofísica Hospitalaria. Además la metodología de cálculo e implementación en diferentes SRD, hace que la incertidumbre del valor calculado pueda en ocasiones ser muy alta (>100\%) y que no sean comparables los valores informados por dos sistemas diferentes para un mismo indicador de dosis. 


\section{Requisitos de los sistemas de registro de indicadores dosimétricos}

La digitalización de la imagen permite recoger, de forma exhaustiva, toda la información que es relevante a la hora de estimar las dosis de radiación recibidas por los pacientes.

Se dispone entonces de un volumen de información que permitirá extraer resultados de dosis a diferentes niveles. En función de cada nivel la recogida de datos debe hacerse de una u otra forma:

- Para el establecimiento de estadísticos locales y su uso o comparación con Niveles de Referencia para Diagnóstico en los procesos de optimización, siendo este el uso principal de estos sistemas.

- Para la elaboración de un historial de indicadores dosimétricos de cada paciente.

- Para poder calcular valores de dosis en órganos o el valor máximo de dosis en la piel de un paciente en un procedimiento intervencionista.

Un sistema de registro y gestión de indicadores dosimétricos debe realizar tres tareas básicas:

1. Recogida de los datos

2. Funcionalidades de análisis y presentación de:

- Estadísticos

- Historiales dosimétricos (opcional)

- Dosimetrías (opcional)

\section{Alarmas}

A continuación se describen las funcionalidades básicas que se deben exigir a un programa de gestión de dosis, así como otras recomendaciones referentes a su implantación.

\subsection{Recogida de datos: integración}

\subsubsection{Modalidades soportadas}

La información dosimétrica que ha de ser gestionada por un SRD requiere que éste sea compatible, como mínimo, con las siguientes modalidades:

- $\quad$ CT. Computed Tomography

- MG. Mammography
- BT. Breast Tomosynthesis

- NM. Nuclear Medicine

- XA. X-Ray Angiography

- CR. Computed Radiology

- DX. Digital Radiography

- PT. Positron Emission Tomography

- IO. Intra-Oral Radiography

- PX. Panoramic X-Ray

Además, se deberá permitir la recepción de datos de todas las modalidades radiológicas soportadas independientemente del fabricante.

\subsubsection{Orígenes de datos}

La recogida de los Indicadores Dosimétricos deberá realizarse preferentemente a través de objetos DICOM RDSR. Sin embargo, para mantener la compatibilidad con modalidades más antiguas que no dispongan de este servicio, es necesario que también permitan otros formatos de datos, incluyendo por lo tanto herramientas para:

- Recepción y extracción de la información de objetos DICOM RDSR.

- Recepción de imágenes DICOM y extracción de la información contenida en los metadatos de las mismas.

- Recepción y extracción de la información de objetos DICOM MPPS.

- Recepción de imágenes DICOM de captura secundaria generadas por la modalidad y extracción de la información a través de reconocimiento óptico de caracteres (OCR) así como de sus metadatos.

Finalmente es necesario considerar que en determinadas modalidades la forma más completa de adquirir los Indicadores Dosimétricos será simultáneamente a través de la combinación de varios de los orígenes de datos mencionados.

\subsubsection{Tipos de datos soportados}

Como se ha descrito en el capítulo tres, para cada una de las modalidades, existen indicadores dosimé- 
tricos específicos y una serie de parámetros técnicos (kVp, mAs, distancia foco-paciente, colimación, filtros utilizados, etc.), necesarios tanto para permitir el cálculo de la dosis a un paciente concreto como para llevar a cabo las tareas de optimización, que deberían ser registrados por el sistema.

\subsubsection{Forma de comunicación con las modalidades}

Los sistemas de registro deberán poder trabajar, en función de las necesidades del centro donde se instalen y de sus infraestructuras, tanto recibiendo pasivamente la información que envíe cada modalidad o el PACS, como interrogando activamente al PACS

Para la mayoría de las modalidades no es necesario que el estudio completo sea enviado al sistema de registro, sino que se envíe solamente el objeto que contenga la información dosimétrica, y así evitar un tráfico de red innecesario.

En aquellas instalaciones en que exista un PACS ya en funcionamiento, será preferible que el sistema de registro de dosis se conecte y recupere la información directamente de éste. De esta manera se simplifica el número de conexiones realizadas (frente a la conexión individual con cada una de las modalidades).

Especial atención merecen los procedimientos intervencionistas. Los equipos al uso instalados a partir del 6 de febrero de 2018, deberán poseer un dispositivo o función que informe al personal sanitario habilitado y a los encargados de los aspectos prácticos de los procedimientos médicos de la cantidad de radiación producida durante el procedimiento y la capacidad de transferir esta información al SRD. A la hora de configurar alarmas, hay que considerar que en este tipo de estudios se pueden alcanzar valores de dosis que superen umbrales de reacciones tisulares. Esto supone que es necesaria la comunicación de esta situación al médico responsable por si procede la inclusión del paciente, en algún protocolo de seguimiento, teniendo en cuenta el periodo de latencia de dichas reacciones. ${ }^{24,25}$

\subsection{Funcionalidades del sistema}

El sistema debería tener las siguientes funcionalidades:

- Permitir definir perfiles de usuario que determinen los derechos de acceso a la información: clínico, técnico, radiólogo, radiofísico, administración y administrador del sistema.
- Consultar los datos dosimétricos de cada paciente por modalidad así como generar un historial dosimétrico global de cada paciente.

- Realizar un análisis estadístico de los datos dosimétricos por modalidad, equipo y protocolo de exploración.

- Visualizar los estudios realizados por cada uno de los equipos conectados y acceder directamente a la información técnica del estudio.

- Generar listados personalizados, permitiendo la exportación de los resultados en formato "hoja de cálculo" o ficheros "csv".

- Generación de estadísticas descriptivas sobre la selección de datos realizada por el usuario por estudio o modalidad.

- Generación de alarmas de dosis personalizables con umbrales de aviso y alarma configurables. Estas alarmas de operación se deberán poder definir para cualquier nivel de información DICOM (pacienteestudio-serie-imagen-evento de irradiación) o combinación de ellos. Asimismo cada alarma deberá poder ser confirmada por los usuarios.

- Disponer de una interfaz de usuario sencilla y homogénea entre las diferentes modalidades, no requiriendo instalación de software específico en los clientes.

- Disponer de una herramienta que permita la búsqueda y filtrado por cualquiera de los parámetros registrados en la base de datos, permitiendo selecciones múltiples y el uso de operadores booleanos.

- Posibilitar el acceso directo (y sin necesidad de realizar el inicio de sesión) desde la aplicación al PACS del centro para revisión de las imágenes clínicas, sin necesidad de salir de un sistema y entrar en el otro.

- Generar de modo automático y bajo petición de un usuario con permisos suficientes, listados de las alarmas validadas, fecha de modificación, motivo y quien las ha modificado y permitir que un usuario administrador pueda revocar la modificación.

- Emitir en algún formato que sea un estándar de facto (tipo PDF) un historial dosimétrico del paciente, así como informes individuales de cada uno de los estudios con indicación expresa de la existencia de indicadores dosimétricos no validados. 
- Posibilidad de calcular el porcentaje de imágenes rechazadas.

El desarrollo o implementación de todas las funciones exigibles a un SRD dependerá de las posibilidades del entorno en el que se integre, historia clínica, PACS, RIS, etc. En cualquier caso el SRD incluirá como mínimo las prestaciones que posibiliten realizar las tareas exigidas en la legislación vigente.

\subsubsection{Validación de los indicadores dosimétricos}

Para desarrollar adecuadamente el proceso de optimización, es imprescindible que la información dosimétrica recogida por cada modalidad sea correcta. En muchas ocasiones es imposible corregir, para obtener los valores adecuados, la información medida o estimada por el equipo. Hay que tener en cuenta además que, actualmente, el rango empleado por los fabricantes para la tolerancia ( $\pm 20 \%$ ), se puede reducir considerablemente en la mayoría de los casos. La EFOMP recomienda que todos los equipos médicos documenten la máxima incertidumbre (IEC 6060126) del indicador dosimétrico representado. ${ }^{27}$

EI SRD deberá por lo tanto ser capaz de aplicar los factores de corrección adecuados en cada modalidad conectada.

Es entonces necesario que cualquier sistema de registro de indicadores dosimétricos permita reflejar claramente este paso de validación junto con la información dosimétrica registrada. Como mínimo se deberá indicar el factor de corrección aplicado a cada indicador dosimétrico individual así como una identificación del especialista en radiofísica responsable de la aplicación de este factor de corrección y el periodo temporal en que se aplica el mismo.

\subsection{Implantación de un SRD}

\subsubsection{Arquitectura del sistema}

El sistema deberá está compuesto por uno o más servidor(es) que se encargue(n) de:

- Soportar los servicios necesarios para la recepción de los indicadores dosimétricos de acuerdo a los orígenes antes comentados.

- Soportar la base de datos que actúe como base del sistema de registro.

- Servir una aplicación web para la administración y configuración del sistema.
- Servir una aplicación web para la interfaz del usuario final.

Estos componentes deberán poder instalarse en un mismo servidor o en varios, permitiendo además estructuras virtualizadas.

\subsubsection{Modalidades y orden de incorporación}

Uno de los objetivos finales de cualquier sistema de registro de indicadores dosimétricos deberá ser mantener un historial del paciente y para ello se deberán conectar todas las modalidades de imagen que hagan uso de radiaciones ionizantes. No obstante, teniendo en cuenta el riesgo radiológico del paciente, se debería comenzar por las prácticas especiales, que engloban a los procedimientos de alta dosis, donde se pueden producir daños por reacciones tisulares, al cribado sanitario y a la pediatría. En este sentido el orden de implantación podría ser:

1. Cardiología y radiología intervencionista (XA).

2. Tomografía computarizada (TC). En este apartado se deberían incluir algunas modalidades de Medicina Nuclear mixtas, al menos el PET/TC (PT/ CT).

3. Mamografía (MG), como principal exploración presente en los programas de cribado sanitario.

4. Pediatría. Requiere un análisis detallado porque podría afectar a prácticamente todas las modalidades (XA, CT, RF, DX y CR) que en algunos casos no permiten disponer de información dosimétrica relevante, por lo que requiere una acotación específica.

5. Radiología de proyecciones sin y con fluoroscopia no intervencionista ( $X A, R F, D X$ y CR), fijos y portátiles. Supone el mayor volumen de equipos y exploraciones, siendo menos relevante en términos de riesgo al paciente. Debería abordarse solamente cuando todo lo anterior está resuelto, además podría alcanzar incluso a la radiología dental, donde la obtención de información dosimétrica en muchos casos no es posible, al igual que en la modalidad CR. 


\section{Verificación (control de calidad) de las prestaciones del sistema de registro de dosis}

\subsection{Definición}

Un software de registro de indicadores dosimétricos es un producto informático capaz de extraer la información dosimétrica disponible en las diferentes modalidades, dentro de un departamento, servicio u organización, que lleve a cabo diagnóstico por imagen empleando radiaciones ionizantes.

Este producto ha de registrar de forma adecuada, (según lo recogido en el capítulo anterior) los parámetros necesarios, en cada modalidad, para que el especialista en Radiofísica Hospitalaria pueda obtener niveles de referencia en diagnóstico, y estimar dosis absorbidas en órganos y dosis efectivas. Además, habrá que tener en cuenta las posibilidades de cada equipo en lo que a producción de informes de dosis se refiere, teniendo presente las prestaciones al respecto en el momento de su adquisición.

\subsection{Procesos que intervienen en la gestión de la dosis}

Podemos separar las tareas de un sistema de registro de dosis en:

- Producción del indicador de dosis en la modalidad.

- Extracción de los indicadores dosimétricos.

- Sistema de gestión de alarmas y de mensajes relacionados.

- Sistema de explotación de los datos.

Cuando el software asociado al sistema de registro permita la estimación de dosis efectiva o dosis en piel, hay que considerar además, en función de las prestaciones de cada software:

- La verificación del algoritmo empleado en el paso de indicador de dosis o técnica radiológica a dosis efectiva.

- La verificación del factor de conversión del indicador de dosis a dosis efectiva, teniendo en cuenta factores modificadores de la edad y, en caso de TC, del maniquí empleado en la estimación del CTDI ${ }_{\text {vol }}$ o DLP para cada región anatómica.
- La posibilidad de corrección en función de la fecha y del kV, del factor de conversión de $P_{K A}$ a dosis efectiva en cada modalidad.

El aseguramiento de la calidad del proceso exige el aseguramiento de la misma en cada una de las partes que lo componen.

Dentro del proceso distinguimos entre pasos asociados a la producción del indicador de dosis, pasos asociados al almacenamiento del indicador de dosis en el sistema de registro y por último los propios del SRD.

\subsection{El Protocolo Español de Control de Calidad en Radiodiagnóstico Revisión 2011 (PECCRX)}

Los aspectos de control de calidad de los dos primeros pasos se encuentran bien definidos en el PECCRX.

\subsubsection{Verificación de los indicadores de dosis en las diferentes modalidades}

- Grafía:

- DG039 Verificación del funcionamiento del sistema de medida o estimación del producto dosis-área.

- Fluoroscopia:

- DE015 Verificación del funcionamiento del sistema de medida o estimación del producto dosis- área.

- DE016 Valor de la dosis al punto de referencia intervencionista.

- Mamografía:

- MD036 Dosis glandular promedio.

- Equipos dentales CBCT-CBVI:

- CB019 Descriptores de dosis.

- Tomografía Computarizada:

- TC020 Índice de dosis en TC (CTDI).

Se pueden añadir además el producto kerma-área en equipos panorámicos dentales y el CTDIvol y DLP en equipos de tomografía computarizada. 


\subsubsection{Control de calidad en PACS}

- PA005 Verificación de la integridad de las cabeceras DICOM.

- PA006 Verificación de la recepción de estudios en el PACS.

- PA007 Verificación de compresión y transmisión de imágenes.

En la actualidad a los tres apartados anteriores cabría añadir "Verificación de la recepción e integridad de los informes estructurados de dosis en el PACS".

\subsubsection{Control de calidad de los sistemas de registro de dosis}

El desarrollo de este apartado podría constituir por sí mismo, en la actualidad, un anexo de un protocolo de control de calidad. Como punto de partida se puede proponer:

- Para cada modalidad conectada, en función del número de estudios analizados, número de registros vs. número de estudios registrables.

- Muestreo (por ejemplo 10 estudios al año por modalidad) de la correcta extracción de la magnitud y del valor asociado en función de la modalidad y de la disponibilidad en cada equipo. (En equipos modernos análisis del SRDR DICOM y en otros de MPPS, cabecera DICOM u OCR).

- Verificación del correcto funcionamiento del sistema de alarmas dosimétricas. Muestreo (por ejemplo 10 estudios al año por modalidad) del correcto funcionamiento de la indicación de superación del límite establecido.

- Verificación del correcto funcionamiento de las estadísticas proporcionadas por el sistema de registro de dosis (pruebas iniciales) y de la correcta exportación de los datos a formatos diversos (xls, csv, mdb, etc).

- Verificación de factores de conversión de indicador de dosis a dosis efectiva o a dosis en órganos. Esta prueba puede ser difícil de llevar a cabo si no se dispone de un software capaz de realizar estas tareas. Se recomienda solicitar al suministrador la documentación necesaria y si es posible verificar, empleando el software adecuado, los factores entregados por el vendedor.
- Cuando el software incorpore esta funcionalidad, verificación del funcionamiento de la corrección del indicador de dosis por $\mathrm{kV}$ y fecha.

\section{Responsabilidades del radiofísico hospitalario en los sistemas de gestión de dosis}

La Directiva 2013/59 EURATOM ${ }^{21}$ pone de manifiesto la necesidad de contar con la participación del especialista en Radiofísica Hospitalaria (Experto en Física Médica) en lo relacionado con las exposiciones médicas. En particular cabe destacar los aspectos relacionados con el artículo 58. En su apartado b dice: "la información relativa a la exposición del paciente sea parte del informe del procedimiento médico-radiológico". En su apartado d dice: "en las prácticas médicas radiológicas, un experto en física médica participe convenientemente, siendo su nivel de implicación proporcional al riesgo radiológico que presenta la práctica" y hace referencia a procedimientos pediátricos, cribado y aquellos que supongan altas dosis de radiación, como la Tomografía Computarizada y la Radiología y Cardiología Intervencionistas. Por otra parte, en el apartado 28) se expone que la Directiva “... debe proponer requisitos más estrictos en cuanto a la información que debe proporcionarse a los pacientes, el registro y la notificación de las dosis de los procedimientos médicos, el uso de niveles de referencia para diagnóstico y la disponibilidad de dispositivos indicadores de dosis...".

Además, en el artículo 56 se afirma que deben "utilizarse y revisarse regularmente los NRD", para proceder con la optimización "sin dilación" en el caso de que "las revisiones locales demuestren que se supera de manera constante los correspondientes NRD (artículo 58). En el artículo 57, en el que se definen las responsabilidades, se afirma que "el experto en Física Médica debe participar en dicho proceso de optimización". De manera similar, en el artículo 83, dedicado exclusivamente al experto en Física Médica, se afirma que éste debe contribuir a la "optimización de la protección radiológica de los pacientes y otras personas sometidas a exposición médica, incluidos la aplicación y el uso de NRD".

Todo ello supone una acción global encaminada a la optimización de las exposiciones médicas a la población utilizando diferentes estrategias. La adopción de requisitos más estrictos en todos los procesos mencionados en la Directiva, ${ }^{21}$ hace necesaria una acción global y coordinada sobre todos ellos pues todos están relacionados y encaminados al objetivo de mejora del proceso de optimización mencionado en el artículo 57 b de la Directiva. 
Un sistema de gestión de dosis ha de ser una herramienta que aporte información útil sobre indicadores dosimétricos y magnitudes dosimétricas de forma que los datos registrados y reflejados en los informes estén validados por los especialistas en Radiofísica Hospitalaria para cada equipo conectado.

La implicación del radiofísico hospitalario, ha de producirse en varias etapas:

- Definición de las especificaciones de compra del software de gestión de dosis.

- Definición del indicador dosimétrico validado que figurará en el informe médico radiológico.

- Validación individual o por bloques del indicador dosimétrico informado.

- Verificación, control de calidad y aplicación de los indicadores de dosis de la modalidad.

- Gestión de alarmas y notificaciones.

- Cálculo, validación e información de la dosis recibida por los pacientes cuando corresponda.

- Control y actualización de los Niveles de Referencia Locales y de los valores típicos de cada modalidad y comparación con los Niveles de Referencia establecidos.

- Formación periódica del personal sanitario receptor de la información generada por el sistema de gestión de dosis y sobre Niveles de Referencia obtenidos.

- Proponer iniciativas para iniciar procedimientos de optimización basadas en los datos proporcionados por los indicadores dosimétricos.

- Realización de informes estadísticos globales sobre los datos aportados por el sistema de gestión de dosis y su impacto en la dosis recibida por los pacientes.

La información extraída de los equipos por el sistema de gestión de dosis ha de procesarse mediante un procedimiento normalizado a fin de que el valor de la magnitud reflejada en el informe médico radiológico no dependa del método utilizado para obtenerla, sino exclusivamente de la forma de realizar el procedimiento y de su complejidad. El radiofísico ha de prestar especial cuidado en el método de verificación de los indicadores dosimétricos y en la aplicación del factor de corrección correspondiente a los indicadores proporcionados por cada equipo antes de que la magnitud quede reflejada en el informe médico-radiológico.
Disponer de indicadores validados por procedimientos normalizados facilita su interpretación, independientemente del profesional que los valore, y permite comparar los Niveles de Referencia Locales de forma más eficaz.

\section{Bibliografía}

1. Brenner D, Elliston C, Hall E, Berdon W. Estimated Risks of Radiation-Induced Fatal Cancer from Pediatric CT. AJR Am. J. Roentgenol. 2001;176(2):289-96.

2. Miglioretti D, Johnson E, Williams A, Greenlee R, Weinmann $S$, Solberg $L$ et al. The Use of Computed Tomography in Pediatrics and the Associated Radiation Exposure and Estimated Cancer Risk. JAMA Pediatr. 2013;167(8):700-7.

3. Thrall J. Radiation Exposure in CT Scanning and Risk: Where Are We?. Radiology. 2012;264(2):325-8.

4. Mettler F, Bhargavan M, Faulkner K, Gilley D, Gray J, Ibbott $G$ et al. Radiologic and Nuclear Medicine Studies in the United States and Worldwide: Frequency, Radiation Dose, and Comparison with Other Radiation Sources-1950-2007. Radiology. 2009;253(2):520-31.

5. Smith-Bindman R, Miglioretti D, Johnson E, Lee C, Feigelson $\mathrm{H}$, Flynn $\mathrm{M}$ et al. Use of Diagnostic Imaging Studies and Associated Radiation Exposure for Patients Enrolled in Large Integrated Health Care Systems, 1996-2010. JAMA. 2012;307(22):2400-9.

6. SPR, AAPM, ACR, ASRT. Image Gently [acceso: 03/10/2019]. Disponible en http://www.imagegently.org/

7. ACR, RSNA, ASRT, AAPM. ImageWisely. [acceso:03/10/2019]. Disponible en http://www.imagewisely.org/.

8. European Commission (EC). Guidance on Diagnostic Reference Levels (DRLs) for medical exposures, Radiation Protection 109. 1999.

9. ICRP. Diagnostic Reference Levels in Medical Imaging: Review and additional advice. ICRP Supporting Guidance 2. Ann ICRP 2001;31(4):33-52.

10. IAEA. Establishing guidance levels In $X$ ray guided medical interventional procedures: A pilot study. Safety Reports Series No. 59. Vienna: International Atomic Energy Agency; 2009

11. IAEA. Diagnostic Reference Levels (DRLS). [Internet]. [Acceso 3/10/2019]. Disponible en: https://www.iaea.org/resources/ rpop/health-professionals/radiology/diagnostic-referencelevels/about-diagnostic-reference-levels

12. Vano E, Miller D, Martin C, Rehani M, Kang K, Rosenstein $M$ et al. ICRP Publication 135: Diagnostic Reference Levels in Medical Imaging. Annals of the ICRP. 2017. 46(1):1-144.

13. NEMA PS3 / ISO 12052, Digital Imaging and Communications in Medicine (DICOM) Standard, National Electrical Manu facturers Association, Rosslyn, VA, USA [Internet]. [Acceso 3/10/2019]. Disponible en: https://www.dicomstandard.org/.

14. Malone J, Guleria R, Craven C, Horton P, Järvinen H, Mayo $J$ et al. Justification of diagnostic medical exposures: some practical issues. Report of an International Atomic Energy Agency Consultation. Br J Radiol. 2012; 85(1013):523-538.

15. Comisión Europea. Dirección General de Medio Ambiente. PR 118: Guía de indicaciones para la correcta solicitud de pruebas de diagnóstico por imagen. Luxemburgo : OFICINA 
DE PUBLICACIONES OFICIALES DE LAS COMUNIDADES EUROPEAS, 2000.

16. Sociedad Española de Radiología Médica. SERAM: Recomendaciones de "no hacer". [Internet] [Acceso: 03/10/2019]. Disponible en: https://seram.es/images/site/doc_seram_ recom_no_hacer.pdf

17. Sistrom C, Dang P, Weilburg J, Dreyer K, Rosenthal D, Thrall J. Effect of Computerized Order Entry with Integrated Decision Support on the Growth of Outpatient Procedure Volumes: Seven-year Time Series Analysis. Radiology.2009; 251(1):147-55.

18. Hricak H, Brenner D, Adelstein S, Frush D, Hall E, Howell $\mathrm{R}$ et al. Managing Radiation Use in Medical Imaging: $\mathrm{A}$ Multifaceted Challenge. Radiology. 2011; 258(3):889-905.

19. International Commission on Radiation Protection (ICRP). Recommendations of the International Commission on Radiological Protection. ICRP Publication 60. 1991. Ann. ICRP 21(1-3).

20. International Commission on Radiation Protection (ICRP). The 2007 Recommendations of the International Commission on Radiological Protection. ICRP 103. Ann ICRP 2007; 37: 2-4. Disponible en: http://www.icrp.org/docs/P103_Spanish. pdf

21. Boletín Oficial del Estado (BOE). DIRECTIVA 2013/59/ EURATOM DEL CONSEJO de 5 de diciembre de 2013 por la que se establecen normas de seguridad básicas para la protección contra los peligros derivados de la exposición a radiaciones ionizantes, y se derogan las Directivas 89/618/ Euratom, 90/641/Euratom, 96/29/Euratom, 97/43/Euratom y 2003/122/Euratom.

22. European Commission (EC). Radiation Protection $n^{\circ} 185$. European Guidelines on Diagnostic Reference Levels for Paediatric Imaging. S.I. : Luxembourg: Publications Office of the European Union, 2018. ISSN 2315-2826.

23. Moore B, Brady S, Mirro A and Kaufman R. Size-specific dose estimate (SSDE) provides a simple method to calculate organ dose for pediatric CT examinations. Med Phys. 2014, 41(7).

24. Rehani MM, Srimahachota S. Skin injuries in interventional procedures. Radiat Prot Dosimetry, 2011;147(1-2):8-12.

25. Balter S, Miller DL. Patient skin reactions from interventional fluoroscopy procedures. American Journal of Roentgenology, 2014;202(4):W335-42.

26. International Electrotechnical Commission (IEC). IEC 60601 - General requirements for basic safety and essential perfomance. 2005.

27. The European Federation of Organisations for MedicalPhysics (EFOMP). EFOMP Guidelines on the transposition of EU BSS art. 60 in national. 2018. 


\section{Callon}

\section{CANON MEDICAL}

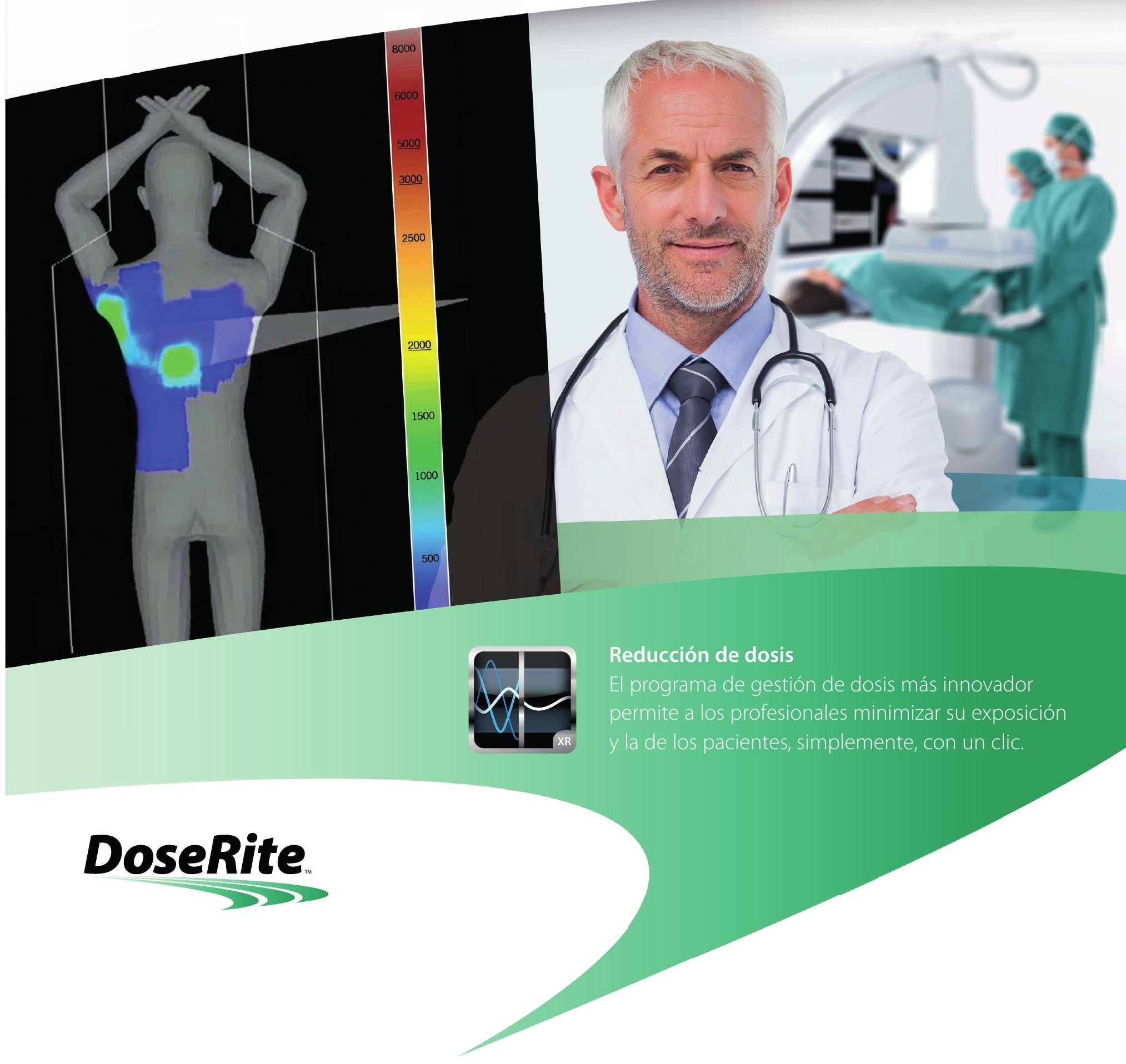

